\title{
Could Investors' Expectations Explain Temporal Variations in Hurst's Exponent, Loci of Multifractal Spectra, and Statistical Prediction Errors? The Case of the S\&P 500 Index
}

\author{
C-René Dominique \\ Formerly Titular Professor of Applied Economics, Laval University, Quebec, Canada \\ E-mail: rdom1@Netzero.net
}

Luis Eduardo Rivera-Solis (Corresponding author)

Professor of Finance, Townsend School of Business, Dowling College

Oakdale, New York 11769, United States

E-mail: riveral@dowling.edu

Received: February 26, 2012

Accepted: March 19, 2012

Published: May 1, 2012

doi:10.5539/ibr.v5n5p8

URL: http://dx.doi.org/10.5539/ibr.v5n5p8

\begin{abstract}
Over the periods 1998-2002 and 2009-2011, the S\&P-500 Index went from persistence to anti-persistence mode, as measured by the Hurst index $\mathrm{H}$. To uncover the reasons that characterize such a change, this paper uses a simple method that consists in treating quasi self-similar segments of the Index as initiators, and then finding appropriate generators with two intervals each to asymptotically model the strange attractor. The multifractal formalism shows that the change in persistence implies a corresponding change in the multifractal spectrum, and an enlargement of the invariant equilibrium set, making a market crash more likely, most probably due to a collapse of investors' expectations. This also means that all statistical predictions made in one mode would have been off by an amount proportional to change in any element of the generalized set of dimensions in the other.
\end{abstract}

Keywords: Persistence, Strange Equilibrium sets, Scaling Exponents, Multifractal Spectra, Generalized Dimensions of order q, Statistical-prediction-error

\section{Introduction}

A Gaussian noise, $X_{t}^{H}$, indexed by the Hurst's exponent $H \varepsilon(0,1)$, may alternate between anti-persistence $(H<1 / 2)$ and persistence $(H>1 / 2)$. Financial time series do frequently undergo such a change, but the reasons have not been identified and studied. To be more specific, consider the available evidence. The computed values of $\mathrm{H}$ vary with methods and/or with time windows (Greene \& Fielitz, 1977; Cutland et al., 1993; Kaplan \& Jay Kuo, 1993; Dominique \& Rivera, 2011, among others), or else, values vary over time (Baraktur et al., 2003; Alvarez-Ramirez, et al., 2008; Preciado \& Morris, 2008). This variability has led theorists to assume that the monofractality of $\mathrm{X}_{\mathrm{t}}^{\mathrm{H}}$ might not be sufficient to characterize random market processes. Consequently, the multifractal formalism was adopted in finance and since has shed much light on the complexity of these processes. But neither have the causes of the alternateness been explored nor has there been so far any policy conclusions drawn from the switch to multifractality.

Multifractality emerged first in statistical physics following on a suggestion of Mandelbrot (1974) to the effect that multifractality might be the best approach to model turbulence so as to account for experimental deviations in the Kolmogorov theory of homogenous and isotropic turbulence. Subsequently, it was shown that indeed many physical processes or quantities do not obey conventional scaling laws. Today the concept of multifractality is routinely used in quantum physics, electrical engineering, meteorology, ecology, geology, medicine, etc. That is to say that it is now well established that these processes exhibit non trivial scaling behavior for different values of the scaling exponent $\mathrm{q}$ $\varepsilon \Re$; implying in the same vein that, differently from monofractals, these processes are generated by multiplicative cascades of random processes.

It is important to note that the study of multifractals on fractal supports is in fact the analysis of invariant sets and measures with multifractal structures. The singularity spectrum of a non-linear process generating an output $\mathrm{f}(\mathrm{x})$, say, with different fractal properties depend on the input conditions. But the output of a dynamical system contains hidden 
information that is revealable by its multifractal spectrum in the forms of, say, a set of dimensions. $Đ(q)=\left\{D_{0}, D_{1}, D_{2}\right.$, $\left.D_{3}, \ldots, D_{\infty}, D_{-1} \ldots, D_{-\infty}\right\} . D_{0}$ is the Hausdorff dimension that measures the exponent of a power law. $D_{1}$ measures how the average information needed to identify an occupied bin scales with a radius size (e) and a probability (p). $\mathrm{D}_{2}$ (due to Grassberger and Procaccia, 1983) examines the number of points needed to generate a representation of a fractal and the number of pairs of points closer than e to each other, etc., up to the Renyi's (1955) generalized dimension of order q that reflects the nature of singularities. Put differently, $\mathrm{D}_{0}$ is a metric indicating how orbits fill up space; $\mathrm{D}_{1}$ and $\mathrm{D}_{2}$ are probabilistic measures assessing the frequency with which orbits visit different parts of an attractor and; in addition, $\mathrm{D}_{2}$, distinguishes between "strange" and "chaotic" attractors. In other words, the set of dimensions allows the reconstruction of the hidden dynamics in conformity with Takens' (1981) Theorem. Thus, detailed analyses of the multifractal structure of an "invariant" set (to be defined shortly) of a chaotic dynamical system provide a more refined description of the chaotic behavior than a description based purely on stochastic characteristics.

The successes of the multifractal formalism in physics and in the other disciplines just mentioned have encouraged its adoption in the study of market price evolution (Halsey, et al., 1986; Fisher, et al., 1997; Muller, et al., 1997; Gopikrishan, 1999; Genyuk, 1999). However, one must bear in mind that invariant sets of most dynamical systems are not self-similar in the strict sense. These sets must be decomposed into subsets supporting a Borel probability measure having some sort of scale symmetry. It is scale symmetries that can reproduce copies of the set on arbitrarily small scales up to a given precision. In such cases, it is important to have on hand processing techniques such as the wavelet transform modulus maxima (WTMM) that can estimate the renormalization parameters from the output as it reveals phase transitions in the singularity spectrum. The absence of WTMM represents a limitation. In applying the multifractal formalism to economics and finance, one should therefore be aware of not only that limitation but of one other caveat. The fact that financial time series are not exactly self-similar is not surprising; no known man-made process is but, in addition, the more uncompetitive financial markets are the noisier are their time series. The consequences of the failure of global self-similarity will be discussed later in our examination of the S\&P-500 Index, and the impacts of noise will be taken up in Appendix A. Our main concern for now, however, is to stress the benefits to be had from the application of the formalism. The most important one for the present purpose is to uncover the characteristics of attractors, which should be important inputs into the decision-making process (vide infra).

The main purpose of this paper is to show that shifts in investors' expectations or behavior might be a compelling factor begetting shifts in persistence of financial time series, which begets shifts in loci of multifractal spectra, which in turn beget errors in statistical predictions. The paper is divided into five parts. Part II is devoted to definitions of terms, concepts, and methodological considerations. Part III discusses the data. Part IV presents the results, which are discussed in Part V.

\section{Definitions of Terms and Method}

The application of the multifractal formalism to financial time series presupposes that the equilibrium sets of prices are closed attracting invariant sets, which are Cantor-like point sets that are either strange or chaotic. But, in discussing such matters, often the differences between deterministic chaos and randomness, for example, or between strange and chaotic attractors, etc, are not always clear or are sometime used as synonyms. Because of the ambiguities associated with definitions of many fractal concepts, it might therefore be useful to begin by defining their meanings in the present context.

Consider a vector field: $\dot{x}=d x / d t=f(x)$, where $f \varepsilon C^{1}(E), E \subset \Re^{n} ; \varphi_{t}(x)$ is the flow of $f$; and $N_{x}(B)$ is a neighborhood of a set $B \subset E$. $B$ is invariant with respect to the flow of $f$ if $\varphi_{t}(x) \subset N_{x}(B)$ at $t \geq 0$ and $\varphi_{t}(x) \rightarrow B$ as $t \rightarrow \infty$. In this case, $B$ is positively invariant with respect to the flow, but $B$ is negatively invariant with respect to the flow if $\varphi_{t}(x) \rightarrow B$ for $\forall \mathrm{t} \leq 0$. Then:

\subsection{Definitions}

Definition 1 (Strange attractor). The attracting set B, just defined, is a strange attractor if it contains:

A countable set of periodic orbits $\left(\Gamma_{p}\right)$ of large periods;

A countable set of non-periodic orbits $\left(\Gamma_{n p}\right)$, and;

\section{A dense orbit.}

The flow $\varphi_{\mathrm{t}}($.$) : \mathrm{E} \rightarrow \mathrm{E}$ or, equivalently a map $\mathrm{h}: \mathrm{E} \rightarrow \mathrm{E}$, is a diffeomorphism of a smooth Riemanian maniforld $\mathrm{E}$, and $\mathrm{B} \subset \mathrm{E}$ is a compact hyperbolic attractor for $\mathrm{h}$. It can then be shown that the evolution of the Lebesque measure on $\mathrm{B}$ converges to the Bowen-Ruelle-Sinai measure, which describes the orbit distribution of points in $\mathrm{N}_{x}(B)$ that are typical with respect to the Lebesque measure. 
Further, if any two arbitrarily close points ( $x, y)$ in $N_{x}(B)$ at $t \geq 0$ become exponentially distant as $t \rightarrow \infty$, then sensitive dependence to initial conditions (SDIC) exists. Then:

Definition 2 (Chaoticity). If the strange attractor of Definition 1 has SDIC, then it is chaotic.

Definitions 1 and 2 are in the sense of Eckmann, and Ruelle (1985).

If now we consider price index as the observable output or an observable signal, $\mathrm{Z}_{\mathrm{t}}$, we have:

Definition 3 (The Process): $Z_{t}=\sum_{i}^{n}\left(b_{i} X_{t}^{H i}\right)$, where $b \varepsilon \Re$, $i \varepsilon n$, is a combination of observed Gaussian processes, each with its own $H$ index, while $X_{t}^{H}$ are unobservable inputs into $Z_{t}$, arriving as "cars" or as "trains" in the sense of Sottinen (2003).

In the literature, $Z_{t}$ is termed: Mixed Fractional Brownian Motion (MfBm) (see, Zili, 2006; Maio, et al., 2008; Thale, 2009). While $X_{t}^{\text {Hi }}$ is the Mandelbrot- van Ness (1968) process (fBm). In general, $X_{t}^{\text {Hi }}$ has the following properties:

Property 1 (Self-Similarity). $X_{t}^{H i}$ and $m^{H i}\left(X_{t}^{H i}\right)$, where $m \varepsilon \Re$, i $\varepsilon n$, have the same probability distribution;

Property 2 (Stationary Increments). Over the interval [t, $s], X_{t}^{\text {Hi }}$ has a normal distribution with zero mean and variance given by $\mathrm{E}\left[\mathrm{X}_{t}^{\mathrm{Hi}}, X_{\mathrm{s}}^{\mathrm{Hi}}\right]=/ t-\left.s\right|^{2 \mathrm{Hi}}$.

The Process of Definition 3, being man-made, lacks global self-similarity, but it has fractal properties including property $2 . \mathrm{X}_{\mathrm{t}}^{\mathrm{Hi}}$, on the other hand, is statistically self-similar, has property 2 and determines the value of $\mathrm{H}_{\mathrm{i}}$. The lack of global self-similarity is carried over to $\mathrm{B}$ and can easily be demonstrated by comparing the values of various elements of the dimension set, $\mathrm{Ð}(\mathrm{q})$. The failure of $Z_{\mathrm{t}}$ on Property 1 is not complete for it is statistically self-similar on segment $\mathrm{i}$. That in itself is not an impediment for the wavelet transform is a function that is equivalent to decomposing the function into elementary space scale. It can be shown that the variance of wavelet coefficients can sufficiently characterize the index $\mathrm{H}_{\mathrm{i}}$ from the slope of the variance plotted as a function of scale in log-log plot (for details, see Flandrin, 1992, 911; Halsey, et al., 1986). The important point to note is that the variability of $\mathrm{H}$ over time is viewed as an indication of the multifractality of $\mathrm{Z}_{\mathrm{t}}$.

\subsection{The Method}

The connection between non-integer fractal dimension and chaos in dissipative continuous dynamical systems results in complex dynamics resulting from the continuous stretching and folding of a bundle of orbits under the action of the flow. In multi-dimensional systems, the geometrical image of their attractors has been shown experimentally to have fractal dimensions between 2 and 3 . In the present case, the set B occupies a small fraction of the embedding space. If we consider its singularity spectrum as its image, then we can work on a one hump map; for the spread of the spectrum $\left(\alpha_{\max }-\alpha_{\min }\right) \varepsilon[0,1]$ and its height is $\mathrm{D}_{0}=\mathrm{f}(\alpha)$. Hence, the procedure adopted for this paper focuses on the image of $\mathrm{B}$, and first considers an output of an unknown system as an "initiator" with a known characteristic, which can either be a generalized "generator" or a measure such as the Hausdorff dimension. The initiator is next divided into two constant bins, while the generator is assigned line segments $e_{i}$ and probabilities $\mathrm{p}_{\mathrm{i}}$. In the third step, the exponent $\mathrm{q}$ is assigned to the probabilities and the exponent $\tau$ is assigned to the support intervals.

Now it is possible to construct the set $\mathrm{Đ}(\mathrm{q})$. Its elements are computed according to Renyi's generalized dimensions. If the above procedure is defective in any way, the magnitudes of the elements of $Đ(q)$ may be trivial. If, on the other hand, it is correct, then $Đ(q)$ must be an ordered set with $\mathrm{D}_{\infty}$ as its minimum element. To be specific:

$$
Đ(q)=\left\{S_{1} \cup S_{2}\right\} \text {, where } S_{1}=\{D(q \geq 0)\}, S_{2}=\{D(q \leq 0)\}
$$

If $Đ(q)$ is divided into two subsets, $S_{1}$ and $S_{2}$ then $D_{\infty}$ is the minimum element of $S_{1}$ and $D_{0}$ is the minimum element of $\mathrm{S}_{2}$. From this line of reasoning, a simple test can be devised in terms of the multifractal spectrum, $f(\alpha)$, to check the validity of the procedure. That is:

Test 1:

i) the function $f(\alpha)$ must have a convex hypograph, and

$$
\text { ii) } D_{0}=\left\{\begin{array}{l}
D_{\max } \text { for } q \geq 0, \forall q \varepsilon \Re \\
D_{\min } \text { for } q \leq 0, \forall q \varepsilon \Re
\end{array}\right.
$$

As in the case of the logistic parabola, the Hausdorff dimension $\left(D_{0}\right)$ can be computed with reasonable precision theoretically and experimentally for each segment $i$ (henceforth $Z_{t}^{i}$ ). Assuming that $Z_{t}^{i}$ is a multifractal, we will select two representative segments that will next be characterized by the two scaling exponents each. This means that the attractor will be asymptotically modeled by the rule of the recursive construction which is a known generator $(\rho)$ with two interval lengths $\left(\mathrm{e}_{1}, \mathrm{e}_{2}\right)$ for each segment, or moving backward from $\mathrm{D}_{0}$ to determine the unknown generator. 
Recalling, in passing, that the rate of return $\pi(\mathrm{P})=\mathrm{f}(\mathrm{P})$ is characterized by a one hump map. Then the logistic parabola, which is prototypical fractal attractor, provides a clue as to the relation between the two interval lengths. From the partition function:

$$
\Sigma_{\mathrm{I}}\left(\mathrm{p}_{\mathrm{i}}^{\mathrm{q}} \mathrm{e}_{\mathrm{i}}^{\tau}\right)=1 \text {, positing } \mathrm{e}_{1}=\left(\rho^{-1}\right), \mathrm{e}_{2}=\left(\rho^{-1}\right)^{2} \text { and } \tau(\mathrm{q})=(1-\mathrm{q}) \mathrm{D}_{\mathrm{q}}
$$

We can derive two equations in quadratic form:

$$
\begin{aligned}
& \mathrm{e}_{1}^{2 \mathrm{Do}}+\mathrm{e}_{1}^{\mathrm{Do}}-1=0 \\
& \mathrm{e}_{1}^{2 \tau}+\mathrm{e}_{1}^{\tau}-1=0
\end{aligned}
$$

Equation (2) is derived from positing $q=0, D_{q}=D_{0}$, and $p_{1}=p_{2}=1 / 2$. Then, $e_{1}{ }^{\text {Do }}=\left[(5)^{1 / 2}-1\right] / 2=G$, where $G$ is the Golden Mean; hence,

$$
\mathrm{D}_{0}=\log \mathrm{G} / \log \mathrm{e}_{1}
$$

From (3), we have:

$$
\mathrm{e}_{1}^{\tau}=\left\{\left[\left(1+4(2)^{\mathrm{q}}\right]^{1 / 2}-1\right\} / 2\right.
$$

then:

$$
\left.\tau=\log \left[\left(1+4(2)^{\mathrm{q}}\right)^{1 / 2}-1\right] / 2\right\} / \log \mathrm{e}_{1}
$$

As the exponent $\tau$ (q) describes the same aspect of the multifractal spectrum, denoted $f(\alpha)$, we have:

$$
\left.\tau /(1-\mathrm{q})=\mathrm{D}_{\mathrm{q}}=\underset{\substack{\lim \mathrm{e} \\ 1} \rightarrow 0}{\log }\left\{\left(1+4(2)^{\mathrm{q}}\right)^{1 / 2}-1\right] / 2\right\} /(1-\mathrm{q}) \log \mathrm{e}_{1} \text {, for } \mathrm{q} \neq 1
$$

Equation (7) is Renyi's $(1955,1970)$ generalized dimensions of order q, which handles every portion of the support of the attractor in a uniform manner and describes the nature of singularities at the same time. It works for all q's, except of course $\mathrm{q}=1$.

For $\mathrm{D}_{1}$, we have

$$
\mathrm{D}_{1}=2 \log 2 / \sum_{1}^{2} \log \left(1 / \mathrm{e}_{\mathrm{i}}\right)
$$

but for $\mathrm{D}_{\infty}$ and $\mathrm{D}_{-\infty}$, it is easier to expand the numerator of (7). That is,

$$
\mathrm{D}_{\mathrm{q}}=-\log 2[\log 2(1+\mathrm{q})] /(1-\mathrm{q}) \log \mathrm{e}_{1}
$$

using $\log _{2}$ and letting $\mathrm{q} \rightarrow \infty$ or $(-\infty)$, we have:

$$
\begin{array}{ll}
\mathrm{D}_{\infty}=\left(-1 / \log _{2} \mathrm{e}_{\mathrm{i}}\right) ; & \mathrm{D}_{-\infty}=\left[-1 / \log _{2}\left(\mathrm{e}_{1}\right)^{2}\right] \\
\lim _{\mathrm{q} \rightarrow \infty} & \lim \mathrm{q} \rightarrow-\infty \\
\mathbf{e} \mathbf{\rightarrow} \mathbf{0} & (\text { e1 } \mathbf{2} \rightarrow \mathbf{0}
\end{array}
$$

The numerator of (4) is a well-known natural sequence. Then if it is $D_{0}$ that is known instead of $\rho$, one may ask what is the implicit generator of any Gaussian process whose Hausdorff dimension is $\mathrm{D}_{0}$ ? Then:

$$
\mathrm{e}_{1}=\log ^{-1}\left[\log \mathrm{G} / \mathrm{D}_{0}\right]
$$

where $\mathrm{e}_{1}$ is from (1). All the D's (except $\mathrm{D}_{1}$ ) can be computed using (7).

The computed values of $\mathrm{D}_{\mathrm{q}}, \forall \mathrm{q}$ must pass Test 1 above. Also, as noted above, the logistic parabola is a prototypical strange attractor that is well-studied. Its Hausdorff dimension derived analytically and numerically, is $0.538 \ldots$ (see Grassberger, 1981; Medio, 1992; Falconer, 2003). In Appendix A, the validity of this method is double-checked against it.

\section{The Data}

The data is the grand Microsoft Excel data set of closing prices of the S\&P-500 Index, sampled daily from January $3^{\text {rd }}$, 1950 to February $28^{\text {th }}, 2011$, from which two appropriate segments were selected for the analysis. The first consists of $2^{10}$ data points, covering the period 1998-2002, when the Index was persistent. The shorter segment consists of $2^{9}$ observations over the period 2009-2011, when the Index was anti-persistent. Both segments were de-trended using logarithmic differences and filtered for white noise and extra noise due to the segmentation, and both met the recommended minimum length $\left(\geq 2^{9}\right)$ for the Wavelet system.

The analysis is done with the Excel SS and the Benoit $\mathrm{TM}_{\mathrm{TM}}$ Wavelet system. The Hausdorff dimension, $\mathrm{D}_{0}$, of segment 1 is $1.390\left(\mathrm{H}_{\mathrm{i}}=0.610 \pm 0.0612\right)$; for segment $2, \mathrm{D}_{0}=1.857\left(\mathrm{H}_{\mathrm{i}}=0.143 \pm 0.0339\right)$. As already noted, one segment shows persistence; while the other indicates that the Index became anti-persistent in recent times. As we will deal with asymptotic values, our calculations will be carried out up to six decimal places for consistency, without however 
making any claim for accuracy beyond that of the exogenously determined Hausdorff dimension. Our results are given below.

\section{The Results}

The Legendre transform does take one from $q$ and $\tau$ to $\alpha$ and $f(\alpha)$, where $\alpha$ is the Lipshitz-Hölder mass exponent and $\mathrm{f}(\alpha)$ is the multifractal spectrum. Thus, $\mathrm{f}(\alpha)$ describes the fractal dimension of a subset having a mass exponent $\alpha$, and gives the same information as $\tau(\mathrm{q})$, since $\alpha(\mathrm{q})=-\mathrm{d} \tau(\mathrm{q}) / \mathrm{dq}, \mathrm{df}(\alpha) / \mathrm{d} \alpha=\mathrm{q}$, and $\mathrm{f}(\alpha)=\mathrm{d} \tau / \mathrm{dq}-\tau(\mathrm{q})$. Put differently, the span of $\alpha$ describes the different subsets of singularities; $\alpha$ is the singularity strength or the local Holder exponent; and $\mathrm{f}(\alpha)$ gives the fractal dimension of the set having Holder exponent $\alpha$; and at $q=0$, one obtains the fractal dimension of the support of the measure. The multifractal information can be presented in more than one form. For the present purpose, however, it is given in terms of $\mathrm{q}$ and $\mathrm{D}_{\mathrm{q}}$ to save space.

Our results are given in Table 1 at the end of the paper. As it can be seen, the right side of Table 1 describes the attractor in anti-persistence mode, while the left side describes it in persistence mode. If the Hurst exponent varies over time, then moving from persistence to anti-persistence, say, implies a rightward translation of the multifractal spectrum. And this has important consequences for market stability, as will be discussed further in the nest section.

Moreover, suppose $D_{q}{ }_{q}^{i}$ is a given value in anti-persistence for a given $q$, while $D_{q}{ }^{j}$ is a persistence value for the same $q$. Then $\mathrm{D}_{\mathrm{q}}{ }^{\mathrm{i}}>\mathrm{D}_{\mathrm{q}}{ }^{\mathrm{j}}$ or $\mathrm{D}_{\mathrm{q}}{ }^{\mathrm{i}} / \mathrm{D}_{\mathrm{q}}{ }^{\mathrm{j}}=v>1$. Therefore, the unknown value $\mathrm{D}_{\mathrm{q}}{ }^{\mathrm{j}} \equiv v^{-1} \mathrm{D}_{\mathrm{q}}{ }^{\mathrm{i}}, \forall \mathrm{q} \varepsilon \Re$. This information represents an economy of time and effort in the assessment of impacts of changes in $\mathrm{H}$.

Having the meaning of these dimensions in mind, it is easily seen that that knowledge increases our understanding of the geometrical structure of the attractor (or the set B) as well as the level of complexity of the flow $\left(\varphi_{t}\right)$; a good example is that $\mathrm{D}_{2}$ distinguishes between deterministic chaos and pure randomness. Moreover, Table 1 shows that despite the Wavelet correction, the self-similarity of the S\&P-500 Index is limited to a given range since $\mathrm{D}_{0} \neq \mathrm{D}_{1} \neq \mathrm{D}_{2}$, implying that the covering of the attractor is neither complete nor quite uniform. As to whether this strange attractor is chaotic or not depends on values of the tuning factor well beyond $r_{\infty}$ (see Appendix A).

Table 1 also shows that a move from persistence to anti-persistence implies an enlargement of the attracting set $\mathrm{B}$ of Definition 1 and a rightward translation of the multifractal spectrum curve. Surely, such changes have important implications for policy decisions. That is, even with an empty noise subset, all statistical predictions made in persistence mode, say, would off by a certain amount when the Index moves to anti-persistence.

Finally, Table 1 shows that the procedure satisfies Test 1 and gives results similar to those of the logistic parabola asymptotically modeled with two different generators with two intervals each. The multifractal spectra of the latter are shown in Appendix A.

\section{Conclusions and Recommendations}

The multifractal formalism is applied to two segments of the S\&P-500 Index. The first segment consists of $2^{10}$ daily observations from 1998 to 2002, while the second examines $2^{9}$ daily observations from 2009 to 2011 . Over the first window, the series showed persistence, while the second showed anti-persistence, as measured by their Hausdorff dimensions. The results appear in Table 1. The main findings are the following:

Over the two segments examined, the S\&P-500 Index was not exactly self-similar as evidenced by differences in metric and probabilistic dimensions. This is not surprising. Natural processes are self-similar only over a given range; man-made processes are even less so.

Despite its monofractals appearance, the finer structures of the Index reveal it to be a multifractal characterized by a multitude of scaling exponents, demonstrating that the multifractal formalism is richer in terms of information.

The Index was asymptotically modeled by two generators $(\rho)$ with two intervals each. The results show that a decrease (increase) in the generator implies an enlargement (compression) and a rightward (leftward) translation of the multifractal spectrum. In other words, when the index shifts from persistence to anti-persistence, the attracting set B of Definition 1, which is a small fraction of the embedding space, increases in size. Amplitudes and orbital periods of both periodic and non-periodic orbits increase. This is consistent with a $1 / \mathrm{f}$ noise regime, which is an indication of complexity that makes market crashes more likely.

Any changes in persistence imply a shift in the multifractal spectrum curve that is proportional to the ratio of the dimensions $\mathrm{D}_{\mathrm{q}}, \forall \mathrm{q} \varepsilon \Re$. Our results show that over the two periods examined the shift from persistence to anti-persistence increases the attracting set by approximately 33.6 per cent. As noted before, the fractal dimensions provide qualitative and quantitative information about both the geometry of the attracting set and the complexity of the flow. Accordingly, all future statistical predictions made during persistence mode (1998-2002) would have been off by 33.6 per cent in anti-persistent mode (2009-2011). 
We apply the present procedure to the logistic parabola. The comparison shows that all the fractal dimensions can be derived from an exogenously determined Hausdorff dimension, and that the change in the multifractal spectrum is equal to the change in dimensions.

As to why an index would swing from, say, persistence to anti-persistence is left for further research. However, there seems to be a positive correlation between periods of economic growth and persistence. One may argue ex hypothesi that the jump from persistence to anti-persistence is due to a collapse of investors' expectations. That assumption is based on the fact that in persistence mode, investors arrive in the market as 'trains' because they hold positive expectations of the immediate and long-terms. The reverse seems to characterize the anti-persistence mode. Under that hypothesis, it is not at all surprising that the Index was strongly anti-persistent over the period 2009-2011, demonstrating thereby that the capital market is after all a reflexive construct.

\section{References}

Alvarez-Ramirez, J., Alvarez, J., Rodriguez, E., \& Fernandez, A. (2008). Time-varying Hurst exponent for US stock markets. Physica A, 1959- 1969.

Baraktur, E., Poor, V. H., \& Sircar, R. K. (2003). Estimating the fractal dimension of the S\&P-500 index using wavelet analysis. E-Quad Paper, Department of Electrical Engineering, Princeton Univ., Princeton, N.J. 08544.

Cutland, N. J., Kopp, P. E., \& Willinger, W. (1993). Stock price returns and the Joseph effect: A fractal version of the Black Scholes model. Progress in Probability, 36, 327-351.

Dominique, C-R., \& Rivera, L. S. (2011). Mixed fractional brownian motion, short and long-term dependence and economic conditions: The case of the S\&P-500 index. International Business and Management, 3, 1-6.

Eckmann, J. P., \& Ruelle, D. (1985). Ergotic theory of chaos and strange attractors. Review of Modern Physics, 57, 617-656. http://dx.doi.org/10.1103/RevModPhys.57.617

Falconer, K. (2003). fractal geometry. New York: John Wiley \& Sons.

Flandrin, P. (1992). Wavelet analysis and synthesis of fractional Brownian motion. IEEE Transactions, 28, 910-917.

Fisher, A. J., Calvet, L. E., \& Mandelbrot, B. B. (1997). Multifractality of deutschmark / US dollar exchange rates. Cowles Foundation Discussion Paper no 1165.

Genyuk, J. (1999). Topics in multifractal formalism: local dimension, gobal scaling. Dept of Mathematics Ohio Unoiversity. Retrieved from www.math.osu.edu/node/19421.

Gopikrishan, P. (1999). Scaling of the distribution of fluctuations in financial market indices. Physica E, 60, 5305-5310.

Grassberger, P. (1981). On the Hausdorff dimension of fractal attractors. Journal of Statistical Physics, 26, 173-179. http://dx.doi.org/10.1007/BF01106792

Grassberger, P., \& Procaccia, I. (1983). Measuring the strangeness of strange attractors. Physica D, 9(1-2), 189-208. http://dx.doi.org/10.1016/0167-2789(83)90298-1

Greene, M. T., \& Fielitz, B. D. (1977). Long-term dependence in common stock returns. Journal of Financial Economics, 4, 339-349. http://dx.doi.org/10.1016/0304-405X(77)90006-X

Halsey, T. C., Jensen, M. H., Kadanoff, L. P., Procaccia, I., \& Shaiman, B. I. (1986). Fractal measures and their singularities: The characterization of strange sets. Physical Review A, 33, 1141-1151. http://dx.doi.org/10.1103/PhysRevA.33.1141

Kaplan, L. M., \& Jay Kuo, C. C. (1993). Fractal estimation from noisy data via discrete fractional Gaussian noise and the Haar Basis. IEEE Transactions, 41, 3554-3562. http://dx.doi.org/10.1109/78.258096

Miao, Y., Ren, W., \& Ren, Z. (2008). On the fractional mixed fractional brownian motion. Applied Mathematical. Science, 35, 1729-1738.

Mandelbrot, B. (1974). Intermittent turbulence in self-similar cascades: Divergence of high moments and dimension of the carrier. Journal of Fluid Mechanics, 62, 331-358. http://dx.doi.org/10.1017/S0022112074000711

Mandelbrot, B., \& van Ness, J. W. (1968). Fractional brownian motions, fractional noises and applications. SIAM Review, 10, 422-437. http://dx.doi.org/10.1137/1010093

Medio, A. (1992). Chaotic dynamics: Theory and applications to economics. United Kingdom: Cambridge University Press. 
Muller, U., Dacorna, M. M., Dave, R. D., Olson, R. B., Pictet, O. V., \& von Weizsacker, J. E. (1997). Volatilities of different time resolution: Analyzing the dynamics of market components. Journal of Empirical Finance, 4, 213-239. http://dx.doi.org/10.1016/S0927-5398(97)00007-8

Preciado, J., \& Morris, H. (2008, Oct.). The varying behavior of U. S. market persistence. Paper presented at the World Congress on Engineering and Computer Science 2008, San Francisco, USA.

Renyi, A. (1955). On a new axiomatic theory of probability. Acta Mathematica Hungaria, 6, 285-335. http://dx.doi.org/10.1007/BF02024393

Renyi, A. (1970). Probability theory. Amsterdam: North-Holland.

Schroeder, M. (2009). Fractals, chaos, power laws. New York: Dover Pub., Inc.

Sottinen, T. (2003). Fractional brownian motion in finance and queuing. (Doctoral dissertation). University of Helsinki, Finland, 2003.

Takens, F. (1981). Detecting strange attractors in turbulence. In D. A. Rand and L. S. Young (Eds.), Dynamical systems and turbulence (pp.366-381). New York: Springer Verlag. http://dx.doi.org/10.1007/BFb0091924

Thale, C. (2009). Further remarks on mixed fractional Brownian motion. Applied Mathematical Sciences, 3, 1-17.

Zili, M. (2006). On the mixed fractional Brownian motion. Journal of Applied Mathematics and Stochastic Analyses, 2006, 1-9. http://dx.doi.org/10.1155/JAMSA/2006/32435

Table 1. The multifractal spectrum of the S\&P-500

\begin{tabular}{lll|lll} 
& $\mathrm{e}_{1}=0.70736$ & $\mathrm{e}_{2}=0.500358$ & \multicolumn{2}{c}{$\mathrm{e}_{3}=0.77172$} & $\mathrm{e}_{4}=0.595551$ \\
\hline $\mathrm{q}$ & $\alpha$ & $\mathrm{D}_{\mathrm{q}}$ & $\mathrm{q}$ & $\alpha$ & $\mathrm{D}_{\mathrm{q}}$ \\
\hline 0 & $\alpha_{0}=1.5017$ & 1.3900 & 0 & $\alpha_{0}=2.005709$ & 1.8570 \\
1 & ---- & 1.334713 & 1 & ---- & 1.783247 \\
2 & --- & 1.287319 & 2 & --- & 1.719921 \\
3 & --- & 1.247567 & 3 & ---- & 1.666641 \\
$:$ & $:$ & $:$ & $:$ & $:$ & $:$ \\
$\infty$ & $\alpha_{\min }=1.0014$ & 1.001400 & $\infty$ & $\alpha_{\min }=1.336900$ & 1.336900 \\
-1 & --- & 1.451489 & -1 & --- & 1.937063 \\
-2 & --- & 1.515938 & -2 & --- & 2.024859 \\
-3 & --- & 1.578455 & -3 & --- & 2.108875 \\
$:$ & $:$ & $:$ & $:$ & $:$ & $:$ \\
$-\infty$ & $\alpha_{\max }=2.00200$ & 2.00200 & $-\infty$ & $\alpha_{\max }=2.674614$ & 2.674614 \\
\hline
\end{tabular}

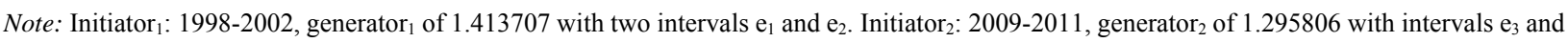
$\mathrm{e}_{4}$. The left side of the table shows the Index in persistence mode; the right side shows the Index in anti-persistence mode.

\section{Appendix A}

Consider the quadratic map:

$$
\mathrm{f}(\mathrm{x})=\mathrm{x}_{\mathrm{t}+1}=\mathrm{r} \mathrm{x}_{\mathrm{t}}\left(1-\mathrm{x}_{\mathrm{t}}\right) \text {, where } \mathrm{x} \varepsilon[0,1] \text {, and } \mathrm{r} \varepsilon(0,4]
$$

Equilibrium values are denoted $x^{*}=(1-1 / r)$, and stability criteria are $\mathrm{df}(\mathrm{x}) / \mathrm{d} \mathrm{x}=\left|\mathrm{f}^{\prime}(\mathrm{x})\right|=\left|\mathrm{r}\left(1-2 \mathrm{x}^{*}\right)\right| \varepsilon[-1,1]$, but $\left|\mathrm{f}^{\prime}(\mathrm{x})\right|=0$ implies a super-stable orbit of the recursive form of $(1 \mathrm{~A})$. For $\mathrm{r}<1, \mathrm{x}^{*}=0$. At $\mathrm{r} \geq 1$, non- zero orbits appear. At $1<\mathrm{r}<3$, there are orbits $\Gamma-2^{\mathrm{k}}, \mathrm{k}=0$. For the purpose, we are interested the behavior of $(1 \mathrm{~A})$ as the parameter $r$ takes on the following values:

$$
\begin{gathered}
\mathrm{r} \approx\left[1+(5)^{1 / 2}\right] \rightarrow \Gamma_{\mathrm{p}^{-}} 2^{\mathrm{k}}, \mathrm{k}=1 \\
\mathrm{r} \approx\left[1+(6)^{1 / 2}\right] \rightarrow \Gamma_{\mathrm{p}}-2^{\mathrm{k}}, \mathrm{k}=2
\end{gathered}
$$

As $r$ is increased further, there are periodic orbits $\Gamma_{\mathrm{p}}-2^{\mathrm{k}}, \mathrm{k}=3,4,5,6, \ldots \mathrm{At}$ :

$$
\mathrm{r}_{\infty} \approx\left[1+(6.602052)^{1 / 2}\right] \rightarrow \Gamma_{\mathrm{np}}-2^{\infty}
$$

orbits become aperiodic, yielding a Cantor set. However, $\mathrm{r}_{\infty}$ does not form an interval. The Lyapunov characteristic exponent (LCE) is zero. Consequently, there is no SDIC and therefore aperiodicity does not imply chaotic behavior as per Definition 2.

The interval $r_{\infty}<r \leq 4$ is referred to as the chaotic regime. However, Medio $(1992,162)$ has shown that, in that window, there exist unstable and stable orbits and chaotic intervals. Even in the interval $\left[1+(8)^{1 / 2}\right] \leq \mathrm{r} \leq\left[1+(8.0738 \ldots)^{1 / 2} \rightarrow \Gamma-3.2^{\mathrm{K}}, \mathrm{k}=1,2,3, \ldots\right.$., termed chaotic by Li and Yorke (1975), there are stable orbits (i. e., LCE $<0$ ), but Medio has also shown that the addition of a small noise is sufficient to change LCE's from negative to positive. Over the window $\left[1+(8.0738)^{1 / 2}<\mathrm{r} \leq 4\right.$, there are only unstable orbits, but unstable orbits or positive LCE's are insufficient to characterize chaos. At $r=4$, all orbits tend to $-\infty$, hence there is SDIC and chaos in our sense. However, the lesson here is that in the presence of noise, all quantitative assessments are invalid. 
Period-doubling is asymptotically self-similar with a universal scaling factor of 2.5029. But the map (1A) is not exactly self-similar. Schroeder (2009) has found that by the fifth iteration, orbits migrate toward a super-stable orbit given by $r_{\infty}$ so that the left half of the unit interval is shrunk by a factor of about 2.5 , while the right half is shrunk by $(2.5)^{2}$. Therefore, the generator is $5 / 2$. On the other hand, analytical and numerical analyses determine the Hausdorff dimension of the quadratic map to be $0.538 \ldots$. (Grassberger, 1981; Medio, 1992; Falconer, 2003). This presents an opportunity to test our procedure.

Schroeder models the attractor with a generator of $5 / 2$. His values, extended to six decimal places by us, appear on the left side of Table $1 \mathrm{~A}$ below. On the other hand, if the exogenously determined value of $\mathrm{D}_{0}$ is $0.538 \ldots$, what is the implicit generator in this case? Using (6) in the text, $\rho=\{[1+$ $\left.\left.(6)^{1 / 2}\right]-1\right\}$. That is the distance between the first non-zero value of $x^{*}$ and the beginning of period 2 orbit. All other D's (for $q \neq 1$ ) are given by Equation (5) in the text. Our results appear on the right side of Table $1 \mathrm{~A}$. As it can be seen, Schroeder's value for $\mathrm{D}_{0}$ is $0.525 \ldots$. , compared with $\mathrm{D}_{0}$ $=0.538 \ldots$. , all D's on the right of Table $1 \mathrm{~A}$ are $\approx 2.4$ percent larger.

Table 1A. The Multifractal Spectrum of the Logistic Map

\begin{tabular}{|c|c|c|c|c|c|}
\hline & $\mathrm{e}_{1}=0.4000$ & $\mathrm{e}_{2}=0.1600$ & & 0.408836 & $\mathrm{e}_{4}=0.167146$ \\
\hline$q$ & $\alpha$ & $\mathrm{D}$ & $\mathrm{q}$ & $\alpha$ & $\mathrm{D}$ \\
\hline 0 & $\alpha_{0}=0.567331$ & 0.525172 & 0 & $\alpha_{0}=0.581042$ & $0.538 \ldots$ \\
\hline 1 & 0.504312 & 0.504312 & 1 & 0.516632 & 0.516632 \\
\hline 2 & --- & 0.486394 & 2 & --- & 0.498278 \\
\hline 3 & ---- & 0.471385 & 3 & ---- & 0.482901 \\
\hline$:$ & $:$ & $:$ & : & $:$ & $:$ \\
\hline$\infty$ & $\alpha_{\min }=0.378234$ & 0.378234 & $\infty$ & $\alpha_{\min }=0.387491$ & 0.387491 \\
\hline-1 & --- & 0.548434 & -1 & --- & 0.561834 \\
\hline-2 & ---- & 0.572787 & -2 & ---- & 0.586782 \\
\hline-3 & ---- & 0.596409 & -3 & ---- & 0.610970 \\
\hline : & $:$ & $:$ & $:$ & : & $:$ \\
\hline$-\infty$ & $\alpha_{\max }=0.756429$ & 0.756429 & $-\infty$ & $\alpha_{\max }=0.774594$ & 0.774594 \\
\hline
\end{tabular}

Note: Schroeder's values with a generator of 2.5 and intervals $\mathrm{e}_{1}$ and $\left(\mathrm{e}_{1}\right)^{2}$ on the left side. And an Implicit Generator of 2.445968 for a Hausdorff Dimension of 0.538 with two intervals $\mathrm{e}_{3}$ and $\left(\mathrm{e}_{3}\right)^{2}$ on the right for an attracting fractal set larger by $\approx 2.44$ per cent. 